\title{
Efficient relay selection and subcarrier assignment in cooperative OFDMA systems with QoS guarantees
}

\author{
Zhanke $\mathrm{Yu}^{1}$, Yanhua Zhang ${ }^{2,3}$, Yan $\mathrm{Guo}^{1,}$, , Mingfang $\mathrm{Ni}^{4}$, and Jun $\mathrm{Zhou}^{4}$ \\ ${ }^{1}$ College of Communications Engineering, PLA Army Engineering University, Nanjing, China \\ ${ }^{2}$ Jiangsu Lightning Protection Center, Nanjing, China \\ ${ }^{3}$ Nanjing University of Information Science \& Technology, Nanjing, China \\ ${ }^{4}$ Guangdong Baiyun University, Guangzhou, China
}

\begin{abstract}
This paper investigates relay selection and subcarrier assignment in cooperative OFDMA systems with considerations of QoS requirements. The joint resource allocation problem is formulated as a $0-1$ integer linear programming problem with the objective of maximizing the system throughput. The efficient joint optimal algorithm is proposed to tackle the problem. By using the penalty function, the complicating constraints are included in the objective function and the special model with a totally unimodular constraint coefficient matrix is constructed that could be solved rapidly as a linear programming with reduced computational complexity. Numerical results show that the proposed algorithm significantly outperforms previous works in terms of both heterogeneous services support and QoS guarantees.
\end{abstract}

\section{Introduction}

Rapid growth of users and explosive growth of new wireless applications require high speed transmission and efficient utilization of system resources. In order to meet this increasing demand, high-spectral-efficiency schemes and aggressive resource reuse strategies are required to ensure prudent use of the scarce radio resources. Cooperative relaying has recently emerged as a promising technique to realize virtual spatial diversity [1-12]. Combined with orthogonal frequency division multiple access (OFDMA), cooperative OFDMA system is considered as the most appropriate technology for modern communication systems [9].

In order to make full use of system resources, various resource management problems have been extensively investigated in recent years. Resource allocation supporting user's QoS requirements for cooperative OFDMA networks was investigated in [1-4]. In [1-3], the authors investigated relay selection and subcarrier allocation in a cooperative OFDMA system with considerations of QoS guarantees and proposed a dual based QoS-aware schedule algorithm. In [4], the authors studied the energy-efficient radio resource scheduling with QoS guarantees in a cooperative OFDMA system. They formulated an optimization

\footnotetext{
* Corresponding author: guoyan_2000@sina.com
} 
problem to maximize the energy efficiency subject to user's QoS requirements and proposed a QoS-aware energy-efficient resource scheduling algorithm based on Lagrangian duality.

In this paper, we investigate relay selection and subcarrier assignment in cooperative OFDMA systems where best effort service (BE) and real-constrained service (RC) are supported simultaneously. The resource allocation problem is formulated as a maximization of the total system throughput by satisfying the users' QoS requirements. It is observed that the problem belongs to the class of integer programming problems, for which an exact solution usually involves an exhaustive search, which is computationally extensive when the number of variables is large. Therefore, we propose the efficient optimal algorithm and include the complicated integer constraints in the objective function as penalty terms and obtain the relaxation 0-1 integer linear problem. After constructing the special model with a totally unimodular constraint coefficient matrix, the relaxed 0-1 integer linear problem can be solved rapidly as linear programming with reduced computational complexity. As the result of optimization, we show numerically that our proposed optimal algorithm significantly outperforms the traditional unconstrained algorithm in terms of both services support and QoS satisfaction.

The rest of the paper is organized as follows. Section 2 introduce the system model and problem formulation. Section 3 then presents joint optimal relay selection and subcarrier assignment algorithm. Section 4 provides the numerical results. Finally, section 5 concludes the paper.

\section{System Model and Problem Formulation}

We consider a single-cell OFDMA relay network with one base station (BS), multiple relay stations (RS) and mobile stations (MS). A slow-fading propagation environment is assumed so that the base station can acquire all channel knowledge perfectly and thus can perform a centralized resource allocation. Let $\left\{r_{0}, r_{1}, \ldots, r_{k}, \ldots, r_{K}\right\}$ denote the $\mathrm{K}$ relay stations, $\left\{m_{1}, m_{2}, \ldots, m_{m}, \ldots, m_{M}\right\}$ denote the M mobile stations, and $\{1,2, \ldots, N\}$ denote the set of subcarriers. The channel coefficients between BS and MS $m$, BS and RS $k$, RS $k$ and MS $m$ on subcarrier $n$ are denoted by $h_{d, m}^{n}, h_{a, k}^{n}, h_{b, k m}^{n}$,respectively.

The BS operates in a time-division-duplexing (TDD) mode and it can communicate with MS either in cooperative mode or non-cooperative mode. For the cooperative mode, we consider two typical types of relay schemes: amplify-and-forward (AF) and decode-and forward (DF). We denote $P_{s, k}^{n}$ as the transmitted power of BS $s$ and RS $k$ on the $n$th subcarrier and denote $P_{r, k m}^{n}$ as the transmitted power of RS $r_{k}$ and MS $m_{m}$ on the same subcarrier, denote $\sigma_{r}^{2}$ and $\sigma_{m}^{2}$ as the noise variances at RS and MS within one OFDM channel, respectively. The instantaneous rate of relay-mobile pair $(k, m)$ on the $n$th subcarrier can be expressed as follow [2]:

$$
\begin{gathered}
c_{k m, A F}^{n}=\frac{1}{2} \log \left(1+P_{s, k}^{n} d_{m}^{n}+\frac{P_{s, k}^{n} a_{k}^{n} P_{r, k m}^{n} b_{k m}^{n}}{1+P_{s, k}^{n} a_{k}^{n} P_{r, k m}^{n} b_{k m}^{n}}\right) \\
c_{k m, D F}^{n}=\frac{1}{2} \log \left(1+\min \left\{P_{s, k}^{n} a_{k}^{n}, P_{s, k}^{n} d_{m}^{n}+P_{r, k m}^{n} b_{k m}^{n}\right\}\right)
\end{gathered}
$$

where $a_{k}^{n}=\left|h_{a, k}^{n}\right|^{2} / \sigma_{r}^{2}, b_{k m}^{n}=\left|h_{b, k m}^{n}\right|^{2} / \sigma_{m}^{2}$, and $d_{m}^{n}=\left|h_{d, m}^{n}\right|^{2} / \sigma_{m}^{2}$.

For the non-cooperative mode, BS transmits directly to the mobile station. Assume the BS transmits with power $P_{s, 0 m}^{n}$ to MS $m_{m}$ on subcarrier $n$, the instantaneous rate can be written as 


$$
c_{0 m}^{n}=\log \left(1+P_{s, 0 m}^{n} d_{m}^{n}\right)
$$

We introduce a binary variable $x_{k m}^{n} \in\{0,1\}$ for all $k, m, n$. When $x_{k m}^{n}=1$, it means that the RS $r_{k}$ is used to relay the signals of MS $m_{m}$ utilizing subcarrier $n$. Otherwise, we have $x_{k m}^{n}=0$. The binary variable $x_{k m}^{n}$ must satisfy the following constraint because each subcarrier can only be used by one user,

$$
\sum_{k=0}^{K} \sum_{m=1}^{M} x_{k m}^{n} \leq 1, \quad \forall n
$$

In this paper, we consider two types of services, best effort service (BE) and realconstrained service (RC), differentiated on the basis of required data rate and bit error rate (BER) criteria. BE service make their best effort to try to deliver a message but bit rate and delivery time cannot be guaranteed, for example, E-mail. RC services are rate-constraint applications such as VoIP and IPTV. Users in the system can be classified into $M_{1} \mathrm{BE}$ users and $M_{2} \mathrm{RC}$ users. For RC users, the QoS requirements can be expressed as

$$
\sum_{k=0}^{K} \sum_{n=1}^{N} c_{k m}^{n} x_{k m}^{n} \geq \bar{c}_{m}, \quad \forall m \in M_{2}
$$

where $\bar{c}_{m}$ is the minimum rate requirement for $\mathrm{RC}$ user.

Our objective is to maximize the system total throughput by jointly optimizing the relay selection and subcarrier assignment. Therefore the optimization problem can be formulated as:

$$
\begin{array}{ll}
\text { (P1) } \max & \sum_{k=0}^{K} \sum_{m=1}^{M} \sum_{n=1}^{N} c_{k m}^{n} x_{k m}^{n} \\
\text { S.t. } & \\
& \sum_{k=0}^{K} \sum_{m=1}^{M} x_{k m}^{n} \leq 1, \quad \forall n \\
& \sum_{k=0}^{K} \sum_{n=1}^{N} c_{k m}^{n} x_{k m}^{n} \geq \bar{c}_{m}, \quad \forall m \in M_{2} \\
& x_{k m}^{n} \in\{0,1\}, \quad \forall k, m, n
\end{array}
$$

\section{Joint Optimal Relay Selection and Subcarrier Assignment Algorithm}

It is observed that problem P1 belong to the class of $0-1$ integer linear programming and it is computationally intractable because of the integrality. Therefore we are interested in solving the problem after relaxing the integrality requirement and exploiting the special network structure of these problems for efficient algorithms in this section.

In order to solve the problem P1, we deal with the QoS constraint matrix (5) by penalty function and construct the following problem.

$$
\begin{aligned}
& \text { (P2) } \max \sum_{k=0}^{K} \sum_{m=1}^{M} \sum_{n=1}^{N} c_{k m}^{n} x_{k m}^{n}+\sum_{m \in M_{2}} \lambda_{m}^{t}\left(\sum_{k=0}^{K} \sum_{n=1}^{N} c_{k m}^{n} x_{k m}^{n}-\bar{c}_{m}\right) \\
& \text { s.t. } \\
& \sum_{k=0}^{K} \sum_{m=1}^{M} x_{k m}^{n} \leq 1, \quad \forall n \\
& x_{k m}^{n} \in\{0,1\}, \quad \forall k, m, n
\end{aligned}
$$

where the nonnegative vector $\lambda^{t}=\left(\lambda_{1}, \lambda_{2}, \ldots, \lambda_{M 2}\right)$ is the penalty vector and $t$ is the number of iterations. 


\subsection{Optimizing penalty vector $\lambda^{t}$}

The QoS constraint matrix (5) is added as penalty terms to the objective in the problem P2. The vector $\lambda^{t}$ is the penalty vector in the penalty terms. If no violation occurs, $\lambda_{i}^{t}$ will be zero and positive otherwise. We optimize penalty vector $\lambda^{t}$ as follows.

$$
\lambda_{m}^{t+1}=\lambda_{m}^{t}+\beta_{m} \Delta \lambda_{m}^{t}, \quad m=1,2, \ldots, M_{2}
$$

where $\Delta \lambda_{m}^{t}$ is the value of violation and $\beta_{m}$ is penalty coefficient for constraint $m . \Delta \lambda_{m}^{t}$ is defined as follows:

$$
\Delta \lambda_{m}^{t}=\max \left(0, \max \left(\bar{c}_{m}-\sum_{k=0}^{K} \sum_{n=1}^{N} c_{k m}^{n} x_{k m}^{n}\right)\right),\left(m=1,2, \ldots, M_{2}\right)
$$

If the inequality is hold, $\max \left(\bar{c}_{m}-\sum_{k=0}^{K} \sum_{n=1}^{N} c_{k m}^{n} x_{k m}^{n}\right) \leq 0$ and $\Delta \lambda_{m}^{t}=0$. If the constraint is violated, $\Delta \lambda_{m}^{t}>0$. Therefore the constraint effect $\lambda_{m}^{t+1}$ and the solution are pushed back towards to the feasible region.

\subsection{Proposed algorithm}

Algorithm 1: Joint optimal relay selection and subcarrier assignment algorithm

Step1: Initialize.

Set the iterate number $t=0$, the maximum iterate number $T=100$, the initial penalty vector $\lambda_{m}^{0}=0,\left(m=1,2, \ldots, M_{2}\right)$ and penalty parameter $\alpha=2$.

Step2: Formulate.

Formulate relay selection and subcarrier assignment problem with QoS guarantees problem as a integer linear programming problem via problem $\mathrm{P} 1$;

Step3: Relax.

Deal with the QoS constraint matrixes by penalty function via problem P2 and relax the integrality constraints to construct relaxed problem with the constraint matrix is totally unimodular via problem P5.

Step4: Solve.

Solve problem P5 by linear programming and obtain variables $x_{k m}^{n}$.

Step5: Evaluate

For $M_{2}$ QoS constraints, compute $\Delta \lambda_{m}^{t}$ according to formula If $\Delta \lambda_{m}^{t}=0,\left(m=1,2, \ldots, M_{2}\right)$, stop with solution $x_{k m}^{n}$, else go to step 6 .

Step6: Penalize and Update.

If $t<T$, compute penalty vector $\lambda_{m}^{t+1}$ according to formula (6). Set $t:=t+1$. Update problem P5, go to step3.

It is observed that the formulated problem P1 belong to the class of integer programming problem, and thus, it is hard to find the optimal solution in a direct approach. In order to solve problem P1, we deal with the QoS constraint matrixes by penalty function and relax the integrality constraints to construct the relaxed problem with constraint matrix is totally unimodular. Solving the relaxed linear programming is much easier than solving 0-1 integer linear programming and make the computation more efficient. The usage of penalty function can guarantee the feasibility of solution and satisfy QoS requirements.

\section{Numerical Results}


To evaluate the performance of our algorithm, numerical results are generated using a simulation scenario. We consider a cooperative OFDMA systems with $\mathrm{N}=64$ subcarriers and a radius of $1 \mathrm{~km}$. Half of the users in the system are assumed to be RC users and the other half are BE users. These $\mathrm{RC}$ users have different rate requirements based on the applications and they are fixed for the whole simulation.

We compare our optimal algorithm with traditional unconstrained algorithm [13] from the perspectives of average sum rate, QoS satisfaction and average running time. Figure 1 shows the average rate obtained by each user for our optimal algorithm and traditional unconstrained algorithm. In the illustrative example, there are $4 \mathrm{RC}$ users with different minimum rate requirements and $4 \mathrm{BE}$ users. The first two $\mathrm{RC}$ users have QoS requirements $\bar{c}_{m}=1$, and the other have higher QoS requirements $\bar{c}_{m}=3$. It is observed that for the unconstrained algorithm, the rates vary a lot between different users and the minimum rate requirements are not fulfilled for users 3 and user 4 which means differentiated services are not supported in the system. But the optimal algorithm satisfy the QoS requirement for all RC users and support all BE users. So, it can be concluded that our proposed optimal algorithm provide not only user satisfaction but also support heterogeneous demand as well.

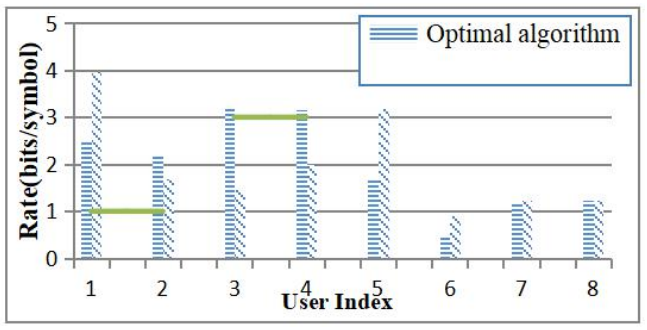

Fig. 1. User achievable rate with $\mathrm{M}=8, \mathrm{~K}=4, \mathrm{~N}=64$, with $4 \mathrm{RC}$ users and $\overline{c_{1}}=\overline{c_{2}}=1, \bar{c}_{3}=\bar{c}_{4}=3$.

Figure 2 shows the average sum rate for the optimal algorithm and the unconstrained algorithm as a function of the number of relays. The optimal algorithm provides slightly lower sum rate compared to the unconstrained algorithm, because the optimal algorithm consider the QoS requirements of RC users. However, the performance gap gradually decreases with an increase in the number of relay stations.

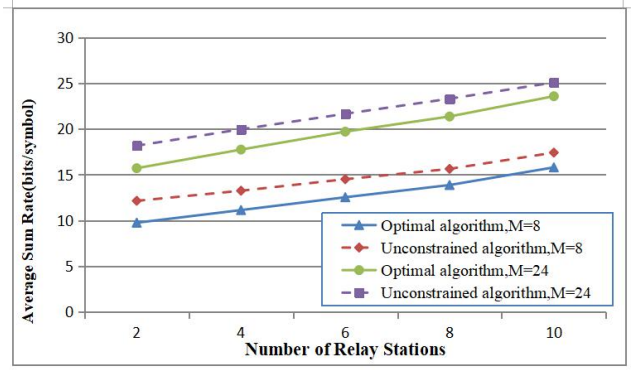

Fig. 2. Average sum rate for 8 and 24 users with different number of relay stations.

\section{Conclusion}

In this paper, we study QoS aware relay selection and subcarrier allocation in cooperative OFDMA systems and formulate the resource optimization problem to maximize total system throughput subject to users' QoS requirements. We propose the efficient joint optimal algorithm, include the complicated integer constraints in the objective function and construct the special model with a totally unimodular constraint coefficient matrix can be solved 
rapidly as linear programming. Numerical results show that the proposed algorithm can support heterogeneous services and satisfy each user's QoS requirements.

\section{Acknowledgment}

This work is supported by the National Nature Science Foundation of China (no. 71401176 and no. 61571463) and supported by Guangdong Science Foundation (no. 2014KQNCX236).

\section{References}

1. Alam M S, Mark J W, Shen X S. Relay selection and resource allocation for multi-user cooperative OFDMA networks[J]. IEEE Transactions on Wireless Communications, 2013, 12(5): 2193-2205.

2. Zhang D, Wang Y, Lu J. QoS aware relay selection and subcarrier allocation in cooperative OFDMA systems[J]. IEEE Communications letters, 2010, 14(4): 294-296.

3. Zhang X, Tao X, Li Y, et al. On relay selection and subcarrier assignment for multiuser cooperative OFDMA networks with QoS guarantees[J]. IEEE Transactions on Vehicular Technology, 2014, 63(9): 4704-4717.

4. Xiao X, Tao X, Lu J. QoS-aware energy-efficient radio resource scheduling in multiuser OFDMA systems[J]. IEEE Communications Letters, 2013, 17(1): 75-78.

5. $\mathrm{Wu} \mathrm{Q}$, Chen $\mathrm{W}$, Tao $\mathrm{M}$, et al. Resource allocation for joint transmitter and receiver energy efficiency maximization in downlink OFDMA systems[J]. IEEE Transactions on Communications, 2015, 63(2): 416-430.

6. Zhang $\mathrm{H}$, Jiang $\mathrm{C}$, Beaulieu $\mathrm{N} \mathrm{C}$, et al. Resource allocation in spectrum-sharing OFDMA femtocells with heterogeneous services[J]. IEEE Transactions on Communications, 2014, 62(7): 2366-2377.

7. Nam C, Joo C, Bahk S. Joint subcarrier assignment and power allocation in full-duplex OFDMA networks[J]. IEEE Transactions on Wireless Communications, 2015, 14(6): 3108-3119.

8. Meng Y, Li J D, Li H Y, et al. Graph-based user satisfaction-aware fair resource allocation in OFDMA femtocell networks[J]. IEEE Transactions on Vehicular Technology, 2015, 64(5): 2165-2169.

9. J. Li, X. Wu, and R. Laroia, OFDMA Mobile Broadband Communications: A Systems Approach. Cambridge U.K.: Cambridge Univ Press, (2013)

10. Mehrjoo M, Awad M K, Dianati M, et al. Design of fair weights for heterogeneous traffic scheduling in multichannel wireless networks[J]. IEEE Transactions on Communications, 2010, 58(10): 2892-2902.

11. Dang W, Tao M, Mu H, et al. Subcarrier-pair based resource allocation for cooperative multi-relay OFDM systems[J]. IEEE Transactions on Wireless Communications, 2010, 9(5): 1640-1649.

12. Lien S Y, Tseng C C, Chen K C, et al. Cognitive radio resource management for QoS guarantees in autonomous femtocell networks[C]//Communications (ICC), 2010 IEEE International Conference on. IEEE, 2010: 1-6.

13. Hsu C N, Su H J, Lin P H. Joint subcarrier pairing and power allocation for OFDM transmission with decode-and-forward relaying[J]. IEEE Transactions on Signal Processing, 2011, 59(1): 399-414. 